\title{
GÉNERO Y FORMACIÓN DEL PROFESORADO: ANÁLISIS DE LAS GUÍAS DOCENTES DEL ÁREA DE DIDÁCTICA DE LAS CIENCIAS SOCIALES ${ }^{1}$
}

\author{
Delfín Ortega Sánchez \\ Universidad de Burgos \\ Joan Pagès Blanch \\ Universitat Autònoma de Barcelona
}

\begin{abstract}
RESUMEN: Con el objeto de conocer el grado de inclusión efectiva de la perspectiva de género en los planes de estudio de Maestro y Maestra de Educación Primaria, este estudio analiza la presencia-promoción o ausencia de este enfoque en las Guías Docentes del área de Didáctica de las Ciencias Sociales de la Comunidad Autónoma de Castilla y León. Con este objetivo, la investigación aplica una metodología de análisis de contenido descriptivo e inferencial mediante el análisis categorizado de los datos devueltos. Los resultados obtenidos informan del mantenimiento general de un currículo docente asimétrico, tendente a la invisibilidad de la educación en y para la igualdad de género, y al fortalecimiento de las relaciones género-poder.
\end{abstract}

PALABRAS CLAVE: Educación Primaria, formación inicial del profesorado, Didáctica de las Ciencias Sociales, perspectiva de género, Comunidad Autónoma de Castilla y León.

\section{GENDER AND TEACHER EDUCATION: ANALYSIS OF THE SOCIAL SCIENCES EDUCATION TEACHERS GUIDES}

\footnotetext{
ABSTRACT: This article analyzes the presence-promotion or absence of the gender approach in the curriculum of the Degree in Teacher of Primary Education. To this end, the paper analyzes the Social Sciences Education Teachers Guides in Castile and Leon. The research applies a methodology of analysis of descriptive and inferential content through the categorized analysis

1 Esta investigación ha sido realizada al amparo del Proyecto I+D MINECO EDU2016-80145-P, Enseñar y aprender a interpretar problemas y conflictos contemporáneos. ¿Qué aportan las Ciencias Sociales a la formación de una ciudadanía global crítica?, coordinado por Antoni Santisteban (Universitat Autònoma de Barcelona), y del Grupo de Innovación Docente de la Universidad de Burgos en Didáctica de las Ciencias Sociales, de las Lenguas y de las Literaturas en la formación inicial del profesorado de Educación Infantil y Primaria, dirigido por Delfín Ortega (Universidad de Burgos).
} 
of the returned data. The results obtained report on the general maintenance of an asymmetric teaching curriculum, tending to the invisibility of the gender equality education and, consequently, strengthening gender-power relations.

KEYWORDS: Primary Education, initial education and training for teachers, Social Sciences Education, gender perspective, Autonomous Community of Castile and Leon.

Recibido: 22/04/2017

Aceptado: 13/07/2017

Correspondencia: Delfín Ortega Sánchez, Universidad de Burgos, Facultad de Educación, C/Villadiego,s/n. 09001 Burgos. Email: dosanchez@ubu.es.

\section{INTRODUCCIÓN}

La investigación confirma la invisibilidad de la experiencia femenina y la ausencia de las relaciones de género en la enseñanza de las Ciencias Sociales (Crocco, 2008; Marolla, 2016; Ortega y Pérez, 2015; Pagès y Sant, 2012; Sant y Pagès, 2011), y en la formación del profesorado (Blanco, 2004; Crocco y Libresco, 2007). Esta ausencia ha venido favoreciendo la ocultación de las diversidades de género y, en consecuencia, el mantenimiento de identidades hegemónicas, tradicionalmente construidas sobre bases androcéntricas (Ortega y Pagès, 2016).

La configuración de lo que podría definirse como identidad/alteridad femenina no sólo responde, como producto cultural e histórico, a condicionantes determinados por la intercomunicación personal y social o a los eminentemente vivenciales y/o experienciales, sino también a los "discursos y realidades que la sociedad [...] presenta y ofrece a hombres y mujeres [...] que condicionará[n], necesariamente, la construcción de la identidad en femenino y masculino, más allá de las respuestas que al mismo puedan dar hombres y mujeres" (Fernández, 2006, p. 33). Es decir, por las representaciones que cada sociedad crea en torno a las definiciones de género.

Los mecanismos para la asignación de identidades femeninas, así como las matrices para su significación subalterna, constituyen los "ejes de la construcción sociocultural de la diferencia, del otro, del colectivo social diferente" (Nash, 2006, p. 40). Las imágenes de alteridad de género y sus estrategias discursivas, por tanto, trascienden el propio sistema de representación para convertirse en prácticas sociales reconocibles.

En la conformación social e histórica de modelos de género deseables (Fernández, 2004), las representaciones sociales y culturales sobre las mujeres toman forma y se consolidan, entre otros discursos posibles -como en el literario o el artístico-, en el propio de la Historia y de su enseñanza. En este discurso, es determinante la generación de narrativas explicativas/legitimadoras del universo simbólico social, donde el paradigma historiográfico tradicional reside como mecanismo cultural de anclaje y permanencia. El reconocimiento de espacios sociales diversos y plurales 
ha de revelar, de esta forma, el carácter constructivo de las identidades de género (social e históricamente determinadas), y ha de apartar la naturalización de la posición subordinada y marginal de las mujeres en las sociedades históricas. En este sentido, el mantenimiento de las estructuras culturales del género, definidas como un "subtipo de estereotipos sociales que incluye lo que se espera que deben 'hacer y ser' las mujeres y los hombres" (Díaz de Greñu y Anguita, 2017, p. 221), derivan en el fortalecimiento del sistema sexo-género, condicionante de los destinos sociales de hombres y mujeres.

Entendiendo la teoría de género como "un saber sobre el que hay que formarse para intervenir las prácticas docentes en el aula" (Palacios, 2009, p. 72), resulta evidente la urgencia de incorporar la formación en igualdad de género en los planes de estudio universitarios (Asián, Cabeza y Rodríguez, 2015), con el fin de adoptar los enfoques de enseñanza-aprendizaje necesarios para la visibilización de las mujeres, la definición de atribuciones identitarias complejas y, en consecuencia, la promoción de la educación para una ciudadanía democrática, crítica e igualitaria.

La inclusión del enfoque de género en el diseño de los planes de estudio de Magisterio y, en particular, en las materias propias del área de Didáctica de las Ciencias Sociales, hace posible el reconocimiento de un tipo de relación de poder, que repercute en el desarrollo profesional del maestro y de la maestra. Se hace necesaria, por tanto, una formación que contribuya a la construcción de una identidad profesional sin sesgos de género, a partir del desarrollo específico de la 'competencia en perspectiva de género'. La perspectiva crítica de esta competencia reside, precisamente, en su postura para la erradicación de los "prejuicios, modelos de comportamiento, creencias y prácticas sexistas, [con el tratamiento de] conocimientos, habilidades y principios éticos y morales no sexistas" (Mora y Pujal, 2009, p. 12).

A pesar del marco legislativo nacional y autonómico vigente ${ }^{2}$, las desigualdades de género siguen estando presentes en la toma de decisiones sobre los contenidos, en la aplicación de las estrategias metodológicas, en la selección de los recursos didácticos, en las relaciones interpersonales, y en la forma en que se distribuye el espacio o se asignan las tareas (Díaz de Greñu y Anguita, 2017). Una realidad que impulsa una formación del profesorado específica y transversal en género (Aguilar, 2013, 2015; Bolaños y Jiménez, 2007; Donoso-Vázquez y Velasco-Martínez, 2013; Romero y Abril, 2008).

No obstante, la preocupación por la integración de la perspectiva de género en la docencia universitaria comienza a recibir una atención significativa. Prueba de ello es la puesta en marcha de iniciativas interuniversitarias como el 'Seminario Internacional de Formación e Investigación e Igualdad de Oportunidades' (Luengo y Rodríguez Sumaza, 2013). Entre sus objetivos, se encuentra la implementación de prácticas educativas capaces de revelar las relaciones de género como un tipo de relación de poder para la promoción del cambio social. En esta línea, también destacan las reflexiones sobre la inclusión de este enfoque en el diseño y en los procesos de

2 Ley Orgánica 1/2004, de 28 de diciembre, de Medidas de Protección Integral contra la Violencia de Género; Ley Orgánica 3/2007, de 22 de marzo, para la igualdad efectiva de mujeres y hombres; Acuerdo 35/2013, de 16 de mayo, de la Junta de Castilla y León por el que se aprueba el Plan Autonómico para la Igualdad de Oportunidades entre Mujeres y Hombres y contra la Violencia de Género de Castilla y León 2013-2018. 
elaboración de los Trabajos Fin de Grado (Aguayo, Freire y Lamelas, 2017; Díez Bedmar, 2017) o en la planificación de las enseñanzas de los títulos de Grado de Maestro y Maestra (Vizcarra et al., 2015).

Con el objetivo de conocer la inclusión real de la perspectiva de género en la adaptación de los estudios de maestro y maestra de Educación Primaria al Espacio Europeo de Educación Superior, esta investigación pretende analizar la presenciapromoción o ausencia de la perspectiva de género en las Guías Docentes del área de Didáctica de las Ciencias Sociales en Castilla y León. De acuerdo con este objetivo, busca determinar el nivel de aproximación a las relaciones de género, y a la educación en y para la igualdad, de las guías vigentes para el curso académico 2016/2017, más allá de potenciales voluntarismos e intereses del profesorado responsable.

\section{Método}

\section{Muestra}

La muestra considerada se compone del total de las Guías Docentes correspondientes al área de Didáctica de las Ciencias Sociales y afines $(\mathrm{N}=19)$ de las universidades públicas (Universidad de Burgos, Universidad de Valladolid, Universidad de León y Universidad de Salamanca) y privadas (Universidad Isabel I de Castilla, Universidad Pontificia de Salamanca y Universidad Católica de Ávila) de la Comunidad Autónoma de Castilla y León. La selección de estas siete instituciones respondió a la necesaria inclusión del título de Grado en Maestro y Maestra en Educación Primaria en su oferta de estudios.

El tipo de muestreo realizado ha sido no probabilístico e intencional o de conveniencia, en tanto que la selección de las instituciones universitarias ha venido determinada en función de la disponibilidad electrónica de las Guías Docentes del área de Didáctica de las Ciencias Sociales en el tiempo de ejecución de la investigación.

Del total de la muestra considerada, un $15.78 \%$ pertenece a la Universidad de Burgos, un $21.05 \%$ a la Universidad de Valladolid, un $15.78 \%$ a la Universidad de León, un $15.78 \%$ a la Universidad de Salamanca, un $10.52 \%$ a la Universidad Pontificia de Salamanca, un $10.52 \%$ a la Universidad Católica de Ávila y un $10.52 \%$ a la Universidad Internacional Isabel I de Castilla (Figura 1).

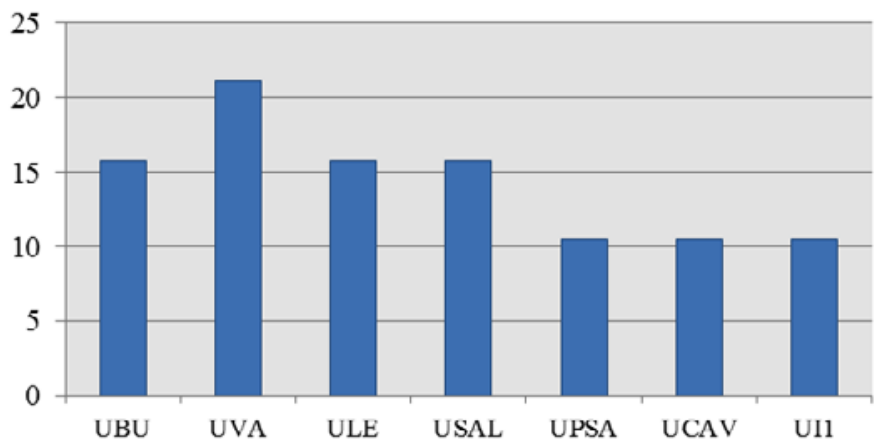

Figura 1. Frecuencias. Guías Docentes seleccionadas por institución 


\section{Instrumento}

El instrumento de vaciado y análisis de datos empleado consistió en una hoja de registro diseñada ad hoc, a partir de la aplicada por Diez et al. (2016), del procedimiento de análisis seguido por Mora y Pujal (2009) y Aguilar (2015), y de la adaptación de las categorías de análisis obtenidas en los resultados del estudio de Fioretti, Tejero y Díaz (2002), categorías definidas en función de la presencia/ausencia de la cuestión de género en la estructura curricular para la formación de educadores y educadoras.

El vaciado de datos ha considerado la justificación educativa de la materia, el desarrollo de contenidos desde la perspectiva de género, la presencia de una finalidad coeducativa en la formulación de objetivos de aprendizaje y de las competencias generales de Título y específicas de materia, el diseño de actividades formativas, los instrumentos de evaluación y las fuentes bibliográficas seleccionadas en la Guía Docente.

\section{Diseño y procedimiento}

La investigación se posiciona en el paradigma crítico, cuyos principios se dirigen hacia la emancipación y transformación social (Giroux, 1999, 2004), la lucha contra la desigualdad y la democratización del currículo (Apple, 1997). Esta perspectiva "busca explícitamente el cambio en las condiciones antidemocráticas en la escuela y la sociedad" (Apple y Beane, 2005, p. 35).

Asimismo, el estudio parte de perspectivas constructivistas, vinculadas a metodologías cualitativas (Bisquerra, 2004; Cohen y Manion, 2002). La metodología empleada en el diseño del instrumento de vaciado de datos y en el análisis de resultados parte de perspectivas descriptivas, interpretativas y críticas. Según esto, la técnica utilizada corresponde al análisis descriptivo e inferencial del contenido manifiesto (Piñuel, 2002). Además, el estudio incorpora análisis estadísticos descriptivos a partir de frecuencias totales, con el objeto de identificar tendencias porcentuales que ayuden a triangular los resultados obtenidos en el análisis del contenido.

Reunidas las unidades de análisis, procedimos a concretar las unidades de registro en palabras clave y raíces semánticas $(*$ mujer y *género / *igual. y *femenin. / *coeduca.), valoradas en su unidad de contexto (justificación, competencias, objetivos, contenidos, actividades, evaluación y bibliografía), y con probabilidad de aparición en alguna de las categorías apriorísticas establecidas. Utilizamos las palabras clave desde su significado contextual y no gramatical, pues "la palabra clave es un símbolo que expresa determinadas actitudes o tendencias" (Ascanio, 2001, p. 41).

Las unidades de registro fueron codificadas mediante dos códigos alfabéticos, en función del carácter de la asignatura, su pertenencia o afinidad al área de conocimiento de Didáctica de las Ciencias Sociales y de su procedencia institucional: B-GA (Básica-General de área)-Institución / B-GN (Básica-General afín)-Institución. Posteriormente, se procedió a cuantificar la frecuencia de aparición de la misma unidad de registro en distintas unidades de análisis. Este dato numérico se incorporó a la codificación alfabética. 
Finalmente, las unidades de registro, ya codificadas alfanuméricamente, fueron categorizadas en uno de los tres enfoques contemplados (categorías apriorísticas): perspectiva excluyente, perspectiva inclusiva interpretativa y perspectiva inclusiva epistemológica (enfoque coeducativo) (Tabla 1).

Tabla 1. Categorización de las unidades de registro

\begin{tabular}{cl}
\hline Categoría & \multicolumn{1}{c}{ Indicador } \\
\hline Perspectiva excluyente & $\begin{array}{l}\text { No se registra la inclusión de la perspectiva de género en } \\
\text { la explicación de la construcción del conocimiento social. } \\
\text { Se constatan tendencias hegemónicas socializadoras y } \\
\text { reproductivas. }\end{array}$ \\
\hline $\begin{array}{c}\text { Perspectiva inclusiva } \\
\text { interpretativa }\end{array}$ & $\begin{array}{l}\text { La presencia de las mujeres y de las relaciones de género en } \\
\text { la construcción del conocimiento social resulta necesaria en } \\
\text { la interpretación de determinados fenómenos sociales. }\end{array}$ \\
\hline $\begin{array}{c}\text { Perspectiva inclusiva } \\
\text { epistemológica (enfoque } \\
\text { coeducativo) }\end{array}$ & $\begin{array}{l}\text { Presencia de las relaciones de género como formas desiguales } \\
\text { de poder y como categoría de análisis de la realidad social. } \\
\text { Se recoge, de forma expresa, la igualdad de trato y de } \\
\text { oportunidades entre hombres y mujeres. }\end{array}$ \\
\hline
\end{tabular}

Para el procesado y análisis de datos, se utilizó el software ATLAS.ti por su capacidad de organización y gestión de información de naturaleza cualitativa.

\section{Resultados}

\section{Inclusión de la perspectiva de género en las materias del área de Didáctica de las Ciencias Sociales y afines}

Vaciados los datos obtenidos, registramos 59 unidades de registro directamente relacionadas con la educación para la igualdad entre mujeres y hombres, y la justicia social. De estas 59 unidades, 16 corresponden a referencias incluidas en asignaturas básicas del área de Didáctica de las Ciencias Sociales y 43 (el 72.88\% de los registros) a otras materias afines vinculadas a la educación para la paz y la igualdad, la educación para la ciudadanía y la educación en valores (Figura 2).

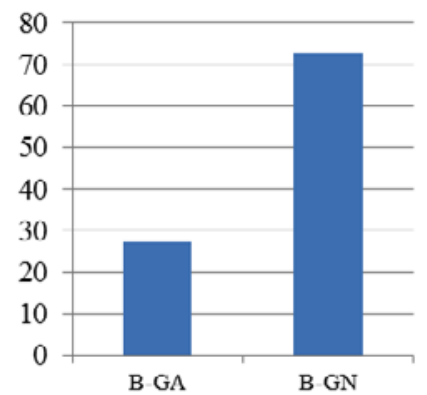

Figura 2. Unidades de registro totales 
Asimismo, se identifican diferencias significativas entre las instituciones universitarias seleccionadas, en función del tipo de asignatura (propia o afín). En efecto, la Universidad Isabel I de Castilla y la Universidad Católica de Ávila reúnen los porcentajes más elevados de las referencias en torno a la igualdad de género en materias propias del área (un $8.47 \%$ y un $6.77 \%$, respectivamente). A estos valores le siguen, por orden decreciente, la Universidad de Burgos y la Universidad de Salamanca (ambas con una frecuencia de un 5.08\%), la Universidad de Valladolid (1.69\%), la Universidad de León (0\%) y la Universidad Pontificia de Salamanca (0\%) (Figura 3).

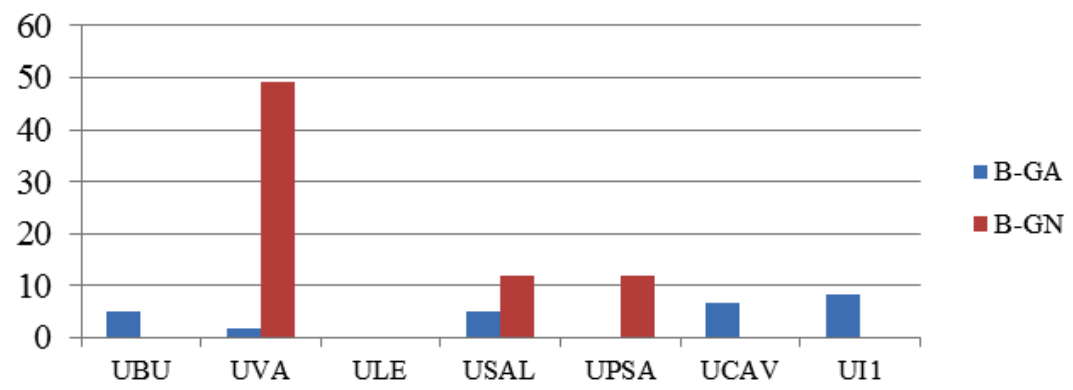

Figura 3. Unidades de registro por institución universitaria

Como puede comprobarse, las referencias a la educación para la igualdad de trato y oportunidades entre hombres y mujeres, a las relaciones de género, o a la erradicación de los estereotipos y sesgos por razón de sexo se registran en materias afines a la Didáctica de las Ciencias Sociales. En este sentido, la Universidad de VaIladolid reúne un importante $49.15 \%$ de las menciones, seguida por la Universidad de Salamanca y la Universidad Pontificia de Salamanca, ambas con un $11.86 \%$ de los registros.

Este desequilibrio evidencia la clara vinculación de la perspectiva de género a áreas disciplinares como la Filosofía o la Pedagogía, y su alejamiento de las finalidades de la enseñanza de las Ciencias Sociales en las acciones docentes para la formación de maestros y maestras.

\section{La igualdad de género en las Guías Docentes del área de Didáctica de las Ciencias Sociales}

Si atendemos al apartado de las Guías Docentes de las asignaturas adscritas al área, las relaciones de género, y la educación en igualdad y para la igualdad entre los géneros mayoritariamente se formulan en las competencias específicas, generales y/o transversales de la materia y, puntualmente, en los objetivos docentes (Figura 4):

Competencia general CG2. Desarrollar un compromiso ético en su configuración como profesional, que potencie la idea de educación integral con actitudes críticas y responsables, garantizando la igualdad de mujeres y hombres, la igualdad de oportunidades, la accesibilidad universal de las personas con discapacidad y los valores propios de una cultura de la paz y de valores democráticos [2. B-GA-UBU]. 
Competencia transversal y específica de módulo BP 23. Analizar e incorporar de forma crítica las cuestiones más relevantes de la sociedad actual que afectan a la educación familiar y escolar: cambios en las relaciones de género e intergeneracionales; multiculturalidad e interculturalidad; discriminación e inclusión social y desarrollo sostenible [1. B-GA-USAL].

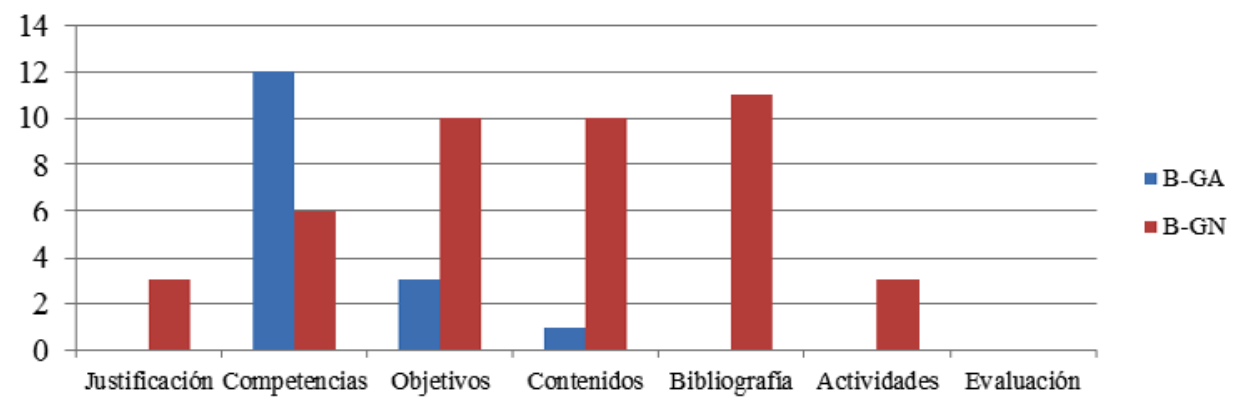

Figura 4. Unidades de registro $B-G A / B G-N$ totales por apartado de las Guías Docentes

No obstante, entre los objetivos docentes, cabe destacar la circunstancial incorporación de la igualdad y la justicia social en torno al fomento de ciudadanías democráticas críticas:

Fomentar la ciudadanía democrática y los valores humanos, la libertad igualitaria, la justicia social, la solidaridad, el sentido crítico, la eliminación de estereotipos y sesgos [1. B-GA-USAL].

Con un solo registro, es anecdótico el tratamiento de la perspectiva de género en los contenidos del módulo de enseñanza y aprendizaje de las Ciencias Sociales:

Vivir en sociedad. Organización y recursos. Valores cívicos. Igualdad de hombres y mujeres [1. B-GA-UI1].

Aunque no puede confirmarse la presencia de perspectivas excluyentes, la incorporación de la perspectiva de género en el diseño de las Guías Docentes seleccionadas tiende a la parcialidad. Esta intencionalidad curricular, concretada en las competencias transversales o específicas ya recogidas en las memorias de los títulos Grado de Maestro y Maestra, no parece devolver, en efecto, evidencias generales de una programación efectiva del enfoque de género.

\section{La igualdad de género en las Guías Docentes de materias afines}

Contrariamente, comprobamos una mayor presencia y una mejor distribución de los registros considerados en materias no adscritas al área de conocimiento. Junto al rechazo a la discriminación en función del sexo, y la educación en y para la igualdad de género, ambas formuladas en competencias y objetivos docentes -tal y como sucede en las asignaturas del área-, estas guías justifican la necesidad educativa del compromiso social del alumnado con la igualdad de género: 
[La asignatura recoge] [...] las competencias referidas a tres leyes que son de obligado cumplimiento: Ley 3/2007 de Igualdad entre Hombres y Mujeres, Ley 51/2003 de No Discriminación y Accesibilidad de las Personas con Discapacidad y la Ley 27/2005 de Cultura de la Paz y que adquieren un especial significado en el caso de la formación de educadores/as y maestros/as de cualquier nivel educativo [1. B-GN-UVA].

Desde una perspectiva inclusiva epistemológica, algunas Guías Docentes adquieren especial importancia en la superación de la tradicional descripción/desarrollo curricular, alcanzando una concepción crítica del currículo con perspectiva de género:

Analizar críticamente los elementos del currículum y los contextos y espacios escolares desde una perspectiva de género [1. B-GN-UVA].

También desde esta perspectiva, es mayoritario el tratamiento de la igualdad de género como contenido:

El respeto a la diversidad debida al sexo, género, raza, orientación sexual, discapacidad, creencias, cultura, etc. [1. B-GN-UVA].

Los problemas derivados de la discriminación por género, raza, orientación sexual, etc. [1. B-GN-UVA].

Igualdad de derechos entre el hombre y la mujer. Pluralidad de creencias, multiculturalismo y tolerancia: Educación para la igualdad entre hombres y mujeres [1. B-GN-USAL].

El valor de la igualdad de oportunidades entre sexos. Valores relacionados. Igualdad y los ámbitos de la vida. Normativa. Informes y estudios. Recursos para favorecer la igualdad [1. B-GN-UPSA].

En este apartado, sólo un registro determina la presencia de las mujeres y de las relaciones de género como contenido necesario en la interpretación de determinados fenómenos sociales (perspectiva inclusiva interpretativa):

El movimiento reivindicador de los derechos de la mujer en los siglos XIX y XX. La igualdad de derechos entre el hombre y la mujer hoy [1. B-GN-USAL].

Es destacable la especificidad con que, de forma puntual, son abordados estos contenidos en el diseño de actividades de formación:

Estudio comparativo de la normativa vinculada a la igualdad, la inmigración o la convivencia. Exposición de conclusiones [1. B-GN-UPSA].

Finalmente, es frecuente la selección bibliográfica de estudios sobre género, igualdad, feminismo, Historia de las Mujeres y coeducación. No obstante, ningún registro sobre evaluación se concreta desde la perspectiva de género.

En función de estos resultados, se comprueba la presencia del género como categoría de análisis de la realidad social. De forma generalizada y homogénea, las Guías Docentes de las materias afines seleccionadas recogen la igualdad de trato y de oportunidades entre hombres y mujeres en la práctica totalidad de los apartados que las conforman.

\section{Discusión Y CONCLUSIONES}

En la línea de recientes investigaciones sobre la presencia/ausencia de la perspectiva de género en las Guías Docentes (Aguilar, 2015; Díez et al., 2016), puede 
afirmarse que, a pesar de las regulaciones vigentes, la inclusión de este enfoque no parece encontrarse entre las responsabilidades formativas de las materias propias del área de la Didáctica de las Ciencias Sociales. Se confirma, en efecto, la presencia de perspectivas parcialmente excluyentes de la cuestión de género para este ámbito de conocimiento, y la divergencia de posicionamientos inclusivos y coeducativos entre universidades públicas y privadas. Según esto, resultan evidentes las dificultades para superar el enfoque androcéntrico en el diseño de las programaciones, y la incoherencia entre los discursos legislativos y las prácticas docentes.

De acuerdo con los resultados obtenidos en otros estudios sobre el pensamiento y posicionamiento de los futuros y futuras docentes acerca de la inclusión de la perspectiva de género en la Historia enseñada (Ortega y Pagès, 2016), se comprueba la persistencia de enfoques metodológicos tradicionales en la enseñanza de las Ciencias Sociales. Esta tendencia motiva la consolidación de imaginarios colectivos/ simbólicos y de representaciones culturales en torno a las mujeres desde este ámbito de conocimiento. En este sentido, Díez Bedmar (2017), en su estudio sobre la inclusión del discurso de género en los Trabajos Fin de Grado y de Máster de Ciencias Sociales, confirma la vinculación de este discurso a una "[...] problemática unida a mujeres, que evidencia posiciones no igualitarias" (p. 112), y el mantenimiento de una "relación directa entre sexo y género", del "feminismo de la diferencia, frente al feminismo de la igualdad" (p. 113).

Pese a la aprobación de regulaciones específicas en materia de género, y la existencia de Planes de Igualdad de Género en las instituciones universitarias, estos no garantizan la incorporación efectiva de la perspectiva de género en las prácticas docentes ni se concretan, de forma visible, en el diseño de las Guías Docentes para la formación del profesorado en la Comunidad Autónoma de Castilla y León. No cabe duda de que, en el ejercicio del compromiso y responsabilidad social de la universidad, "estamos ante el momento idóneo para estimular una formación de carácter transversal prestando especial atención a la perspectiva de género dentro de las competencias del alumnado en las distintas disciplinas de las nuevas titulaciones" (Aguayo, Freire y Lamelas, 2017, p. 15).

Como demuestra la literatura científica en el ámbito de la formación del profesorado (Díaz de Greñu y Anguita, 2017), el mantenimiento de estereotipos y sesgos de género en los discursos y prácticas del profesorado refuerza el sistema sexo-género y, consecuentemente, la desigualdad. En este aspecto, la responsabilidad del área de Didáctica de las Ciencias Sociales en contribuir a la comprensión de sociedades cada vez más plurales e inclusivas necesariamente pasa por la inclusión del género como categoría de análisis (García y Peinado, 2015). La construcción y enseñanza de un conocimiento social integral ha de responder a uno de los problemas sociales más urgentes en el conjunto de preocupaciones investigadoras y docentes del área y del ámbito educativo.

Desde esta perspectiva, siguen siendo necesarias investigaciones que atiendan al análisis de los discursos emergidos de la práctica docente en torno al género, a la reflexión crítica sobre los planes de formación del profesorado y a la transformación del propio currículo docente, a partir de revisiones curriculares como las realizadas por Lerner (1981) y McIntosh (1983). 
La adopción de enfoques coeducativos, y la promoción de una educación en y para la igualdad de género, implican transformar el currículo docente tradicional, con el propósito de superar las bases constructivas androcéntricas del conocimiento histórico y social (Ortega y Pérez, 2015), y de incorporar 'miradas polisémicas' en la comprensión e interpretación de la realidad social.

Aún hoy es frecuente reconocer en otros discursos de comunicación social, como el publicitario o el artístico, una clara imaginería de patrones culturales de género incuestionables, pretendidamente identitarios. La superación o relativización contra-socializadora de dichos patrones necesariamente ha de transitar por la problematización de los contenidos curriculares (Thornton, 2005) y, en atención a la propia naturaleza del conocimiento social, por la incorporación de la perspectiva de género en la enseñanza de las Ciencias Sociales (McIntosh, 2005). En efecto, el reflejo del universo social, obligadamente diverso y heterogéneo, así como el cambio metodológico, constituyen los pilares básicos para contraer compromisos sociales, para la participación ciudadana crítica y responsable, y para la comprensión de los mecanismos de poder, en torno a las relaciones culturales de género. No basta, por tanto, la inclusión o la sustitución de hombres por mujeres o por los otros y otras; es preciso un cambio discursivo (Sánchez, 2002; Crocco, 2008; Pagès y Sant, 2012), accesible al empleo de los estudios de caso, al trabajo de la empatía histórica o al manejo e interpretación de fuentes literarias, documentales o iconográficas en el aula de Ciencias Sociales (Fernández, 2001; 2006). Esto es, la superación de una enseñanza de las Ciencias Sociales excluyente e individualista, capaz de contemplar la transferencia de la investigación histórica con perspectiva de género (García, 2016).

La dirección hacia la transformación y el cambio social en la enseñanza de la Historia y de las Ciencias Sociales, y en la formación del profesorado, se encontraría en la priorización de conceptos clave y en la reformulación de las finalidades atribuidas a dicha enseñanza (Barton y Levstik, 2004). Estos conceptos habrían de partir de la multiplicidad de perspectivas, la democracia, la diversidad, la globalización o la identidad/alteridad, entre otros (Banks, 2004). En definitiva, la adopción de enfoques orientados a la consolidación de una ciudadanía global, inclusiva y diversa, donde el desarrollo del pensamiento crítico y social, y el reconocimiento de identidades de género complejas y múltiples, supongan uno de sus ejes fundamentales.

La erradicación de las desigualdades de género precisa de la integración de todas las voces que han construido y construyen el conocimiento social, y de la superación de los estereotipos de género del profesorado en activo y en formación (Díaz de Greñu y Anguita, 2017). En esta inclusión, coincidimos con Marolla (2016) en afirmar que la visibilización de las mujeres en la enseñanza de la Historia y de las Ciencias Sociales ha de plantearse desde una perspectiva crítica y no aditiva.

La detección diagnóstica de carencias formativas del y la docente, y la incorporación de formación en género en los planes de estudio de Magisterio, favorecerían la transformación de las prácticas docentes para alcanzar modelos sociales verdaderamente inclusivos, plurales y diversos. 


\section{REFERENCIAS BIBLIOGRÁFICAS}

Aguayo, E., Freire, M. ${ }^{a}$ P. y Lamelas, N. (2017). Incorporación de la perspectiva de género en el TFG: una experiencia en el Grado en Economía de la USC. Revista Complutense de Educación, 28(1), 11-28.

Aguilar Ródenas, C. (2013). Género y formación crítica del profesorado: una tarea urgente y pendiente. Revista Interuniversitaria de Formación del Profesorado, 27(3), 177-183.

Aguilar Ródenas, C. (2015). Igualdad, género y diversidad sexual en la Formación Inicial de Maestro/a en la Universidad Jaume I (UJI). Temas de educación, 21(1), 77-96.

Apple, M. y Beane, J. A. (2005). Escuelas democráticas. Madrid: Morata.

Apple, M. (1997). Teoría crítica y educación. Buenos Aires: Miño y Dávila Editores.

Ascanio, A. (2001). Análisis de contenido del discurso político. Caracas, Venezuela: Equinoccio. Ediciones de la Universidad Simón Bolívar.

Asián, R., Cabeza, F. y Rodríguez Sosa, V. (2015). Formación en Género en la Universidad: ¿Materia de asignaturas específicas o de educación transversal? Revista Historia de la Educación Latinoamericana, 17(24), 35-54.

Banks, J. A. (Ed.) (2004). Diversity and citizenship education: Global perspectives. San Francisco: Jossey-Bass.

Barton, K. C. y Levstik, L. S. (2004). Teaching history for the common good. Mahwah, New Jersey: Routledge.

Bisquerra, R. (Ed.). (2004). Metodología de la investigación educativa. Madrid: La Muralla.

Blanco, P. (2004). La perspectiva de género, una necesidad en la construcción de la ciudadanía. Algunas actividades en la formación del profesorado. En I. M. ${ }^{a}$ Vera y D. Pérez i Pérez (Eds.), Formación de la ciudadanía: las TICs y los nuevos problemas (pp. 665-673). Alicante: AUPDCS.

Bolaños, L. M. y Jiménez Cortés, R. (2007). La formación del profesorado en género. Revista de Investigación Educativa, 25(1), 77-95.

Cohen, L. y Manion, L. (2002). Métodos de investigación educativa. Madrid: La Muralla.

Crocco, M. S. y Libresco, A. S. (2007). Gender and social studies teacher education. En D. Sadker y E. Silber (Eds.), Gender and teacher education: Exploring essential equity questions (pp. 109-164). Mahwah, NJ: Erlbaum.

Crocco, M. S. (2008). Gender and sexuality in the social studies. En L. S. Levstik y C. A. Tyson (Eds.), Handbook of Research in Social Studies Education (pp. 172-196). New York: Routledge.

Díaz de Greñu, S. y Anguita, R. (2017). Estereotipos del profesorado en torno al género y a la orientación sexual. Revista Electrónica Interuniversitaria de Formación del Profesorado, 20(1), 219-232.

Díez Bedmar, M. a del C. (2017). "Con y para la sociedad". Análisis del discurso de género de alumnado en Trabajos Fin de Grado/Máster de Ciencias Sociales. En R. Martínez Medina, R. García-Morís y C. R. García Ruiz (Eds.), Investigación en Didáctica de las Ciencias Sociales. Retos, preguntas y líneas de investigación (pp. 102-114). Córdoba: Universidad de Córdoba-AUPDCS. 
Díez Ros, R., Aguilar Hernández, M. M., Gómez Trigueros, I. M. a , Lozano Cabezas, I., Iglesias Martínez, M. J., Pérez Castelló, T. D. y Vera Muñoz, M. a I. (2016). Inclusión de la perspectiva de género en las guías docentes de asignaturas de Didáctica. En M. ${ }^{a}$ T. Tortosa Ybáñez, S. Grau Company y J. D. Álvarez Teruel (Coords.), XIV Jornadas de Redes de Investigación en Docencia Universitaria. Investigación, innovación y enseñanza universitaria: enfoques pluridisciplinares (pp. 2665-2679). Alicante: Universidad de Alicante.

Donoso-Vázquez, T. y Velasco-Martínez, A. (2013). ¿Por qué una propuesta de formación en perspectiva de género en el ámbito universitario? Profesorado. Revista del Currículum y Formación del Profesorado, 17(1), 71-88.

Fernández Valencia, A. (2001). Las mujeres en la enseñanza de las Ciencias Sociales. Madrid: Síntesis.

Fernández Valencia, A. (2004). Las mujeres como sujeto histórico: género y enseñanza de la historia. Didáctica de las Ciencias Experimentales y Sociales, 18, 5-24.

Fernández Valencia, A. (2006). La construcción de la identidad desde la perspectiva de género. Íber. Didáctica de las Ciencias Sociales, Geografía e Historia, 47, 33-44.

Fioretti, S. R., Tejero, G. y Díaz. P. (2002). El género: ¿un enfoque ausente en la formación docente? La Aljaba, 7(2), 171-186.

García Luque, A. (2016). Incorporar la perspectiva de género en la enseñanza-aprendizaje de la historia: un desafío didáctico y formativo. Revista PH, 89, 147-149.

García Luque, A. y Peinado, M. (2015). LOMCE: ¿es posible construir una ciudadanía sin la perspectiva de género? Íber. Didáctica de las Ciencias Sociales, Geografía e Historia, 80, 65-72.

Giroux, H. (1999). Pedagogía crítica como proyecto de profecía ejemplar: cultura y política en el nuevo milenio. En F. Imbernón (Coord.), La educación en el siglo XXI. Los retos del futuro inmediato (pp. 53-62). Barcelona: Graó.

Giroux, H. (2004). Teoría y resistencia en educación. Una pedagogía para la oposición. México D.F.: Siglo XXI Editores.

Lerner, G. (1981). The Majority Finds Its Past: Placing Women in History. New York: Oxford University Press.

Luengo, T. y Rodríguez Sumaza, C. (2013). Construyendo redes para una cultura de la equidad. Enfoque de género y universidad. Revista Electrónica de Psicología Iztacala, 16(2), 636-657. Recuperado de: http://revistas.unam.mx/index.php/repi/ article/view/39974/36375.

Marolla, J. P. (2016). La inclusión de las mujeres en la enseñanza de la Historia y las Ciencias Sociales. Estudio colectivo de casos en las aulas chilenas sobre sus posibilidades y limitaciones. (Tesis Doctoral). Universitat Autònoma de Barcelona. Recuperada de: http://www.tdx.cat/handle/10803/399346.

McIntosh, P. (1983). Interactive Phases of Curricular Re-Vision: A Feminist Perspective. Wellesley, Massachusetts: Wellesley College.

Mclntosh, P. (2005). Gender perspectives on educating for global citizenship. En N. Noddings (Ed.), Educating Citizens for Global Awareness (pp. 22-39). New York: Teachers College Press. 
Mora, E. y Pujal, M. (2009). Introducción de la perspectiva de género en la docencia universitaria. II Congreso Internacional 'Claves para la implicación de los estudiantes en la Universidad. Girona: Universitat de Girona. Recuperado de: http:// hdl.handle.net/10256/2017.

Nash, M. (2006). Identidades de género, mecanismos de subalternidad y procesos de emancipación femenina. Revista CIDOB d'Afers Internacionals, 73-74, 39-57.

Ortega, D. y Pagès, J. (2016). Deconstruyendo la alteridad femenina en la enseñanza de la historia escolar: Representaciones sociales del profesorado de Educación Primaria en formación. En C. R. García Ruiz, A. Arroyo y B. Andreu (Eds.), Deconstruir la alteridad desde la Didáctica de las Ciencias Sociales. Educar para una ciudadanía global (pp. 184-193). Las Palmas de Gran Canaria: Universidad de Las Palmas de Gran Canaria-AUPDCS.

Ortega, D. y Pérez, C. (2015). Las mujeres en los libros de texto de Ciencias Sociales de $1^{\circ}$ de E.S.O. En A. M. ${ }^{a}$ Hernández Carretero, C. R. García Ruiz y J. L. de la Montaña (Eds.), Una enseñanza de las Ciencias Sociales para el futuro: Recursos para trabajar la invisibilidad de personas, lugares y temáticas (pp. 943-951). Cáceres: Universidad de Extremadura-AUPDCS.

Pagès, J. y Sant, E. (2012). Las mujeres en la enseñanza de la Historia: ¿hasta cuándo serán invisibles? Cad. Pesq. Cdhis, Uberlândia, 25(1), 91-117.

Palacios, L. (2009). Epistemología y pedagogía de género: el referente masculino como modo de construcción y transmisión del conocimiento científico. Horizonte Educacionales, 14(1), 65-75.

Piñuel, J. L. (2002). Epistemología, metodología y técnicas del análisis de contenido. Estudios de Sociolingüística, 3(1), 1-42.

Romero Díaz, A. y Abril, P. (2008). Género y la formación del profesorado en los estudios de Educación Infantil. Revista Electrónica Interuniversitaria de Formación del Profesorado, 11(3), 43-51.

Sánchez Bello, A. (2002). El androcentrismo científico: el obstáculo para la igualdad de género en la escuela actual. Educar, 29, 91-102.

Sant, E. y Pagès, J. (2011). ¿Por qué las mujeres son invisibles en la enseñanza de la Historia? Revista Historia y Memoria, 3, 129-146.

Thornton, S. (2005). Incorporating internationalism into the social studies curriculum. En N. Noddings (Ed.), Educating citizens for global awareness (pp. 81-92). New York: Teachers College Press.

Vizcarra, M. T., Nuño, T., Lasarte, G., Aristizábal, M. P., y Álvarez Uria, A. (2015). La perspectiva de género en los títulos de Grado en la Escuela Universitaria de Magisterio de Vitoria-Gasteiz. REDU. Revista de Docencia Universitaria, 13(1), 297-318. 